\title{
The role of the hepatology nurse in the difficult-to-treat hepatitis $\mathrm{C}$ population
}

\author{
Jo-Ann Ford RN MSN ${ }^{1}$, Cindy Cheong-Lee RN²
}

$\mathrm{C}$ vurrently, the primary risk factor for hepatitis $\mathrm{C}$ virus (HCV) is injection drug use; it is estimated that $80 \%$ to $95 \%$ of intravenous drug users (IDUs) are infected or will become infected with HCV in the near future (1). This marginalized group in our society is well recognized to be at risk for alcoholism, psychiatric illness and psychosocial instability $(2,3)$, and historically, they have not been considered viable candidates for HCV treatment. In this issue of The Canadian Journal of Gastroenterology, Moirand et al (pages 355-361), in their paper titled "Determinants of antiviral treatment initiation in a hepatitis $\mathrm{C}$-infected population benefiting from universal health care coverage", retrospectively reviewed HCV treatment and the determinants of antiviral treatment initiation in a vulnerable, urban, inner-city population. The authors evaluated the chart documentation of 3300 patients and identified 378 patients eligible for HCV antiviral therapy. Of the 378 eligible patients, 143 were offered and initiated treatment, while 95 were offered but rejected treatment. Variables that influenced treatment included a resolute request for treatment, participation in methadone maintenance therapy, younger age and past alcohol use. Factors that negatively influenced treatment included current intravenous drug use, alcoholic liver damage on biopsy, precarious or unspecified housing, current depression and personality disorders. An important observation to note from the paper by Moirand et al is that of the patients considered medically eligible for treatment, only $63 \%$ were offered therapy, and of these, only $60 \%$ accepted (ie, less than $40 \%$ of the medically eligible cohort). This suggests that potentially viable patient populations may not be receiving treatment. The work of Moirand et al is consistent with other studies that reported successful treatment of IDUs provided that they were closely supervised. Backmund et al (4) reported on the treatment of 50 IDUs with interferon or interferon and ribavirin combination therapy. The rate of sustained virological response (SVR) was 36\% and was not significantly different between those patients who relapsed to drug use and those who did not. Van Thiel et al (1) also demonstrated that the SVR in IDUs was similar to that in non-IDUs. They also reported that the recent use of intravenous drugs or continued methadone use during treatment did not impair the SVR in comparison with non-IDUs.

Unfortunately, in today's world, the number of treated HCV patients needs to increase dramatically or we will not be able to prevent new infections or reduce the burden of end-stage liver disease. Clearly, we can no longer ignore vulnerable populations, and effective interventions must be in place to meet this challenge. Moirand et al acknowledge the need for a multidisciplinary approach to the management of patients infected with $\mathrm{HCV}$, including the potential role of community organizations, IDU peer groups and social support. Although it was not elaborated on, a team approach must incorporate the knowledge, skills and expertise of the hepatology nurse.

The Canadian Association of Hepatology Nurses (CAHN) was established in November 1999. The genesis of creating a professional national nursing association in hepatology occured in January 1999, when over 30 hepatology nurses from across Canada had the unique opportunity of coming together for a meeting and realized the need for creating a nursing hepatology organization. In February 2000, the first executive was elected, with Colina Yim as President. The CAHN is currently an associate member organization of the Canadian Nurses Association. The membership consists of over 150 registered nurses from across Canada whose clinical practice, research, administration, education or interest resides in the domain of hepatology. The mission of the CAHN is to advance the quality of nursing practice, and to promote advanced clinical and research nursing practice in hepatology across Canada. The CAHN's mandate includes the maintenance and improvement of the qualifications and standards of hepatology nurses. The CAHN promotes educational opportunities for its members both on a national and a local level. Annually, the CAHN holds an education conference in conjunction with its annual general meeting. This meeting has, over the past few years, been held in conjuction with the Canadian Association for the Study of the Liver. Local workshops are supported across the country with topics that include critical appraisal of literature, management of psychological side effects, and the natural history of hepatitis B and its treatment. The organization provides a network in which its members can exchange information as well as gives the opportunity to share the best practices and guidelines on prevention, care, treatment and support. Our newsletters include journal review articles, information on upcoming meetings, highlights from conferences and latest study results. The Web site, which is in the process of being redeveloped (www.cahn.ca), provides membership with access to documents such as the Canadian Consensus Guidelines, as well as the administration and monitoring of erythropoietin guidelines. In the near future, it will provide a slide kit that can be used to educate novice nurses on viral hepatitis. In addition, standards of practice for hepatology nurses have ben approved and will be posted on the CAHN Web site (5). The

${ }^{1}$ Canadian Association of Hepatology Nurses; ${ }^{2}$ BC Hepatitis Program, Vancouver, British Columbia

Correspondence: Ms Jo-Ann Ford, Canadian Association of Hepatology Nurses, 5153 - 2775 Laurel Street, Vancouver, British Columbia V5Z 1 M9. Telephone 604-875-5705, fax 604-875-5221, e-mail joann.ford@vch.ca

Received for publication November 14, 2006. Accepted November 22, 2006 
purpose of the standards document is to provide a basis for the evaluation and guidance of professional, ethical and nursing practice in hepatology. The standards encompass the hepatology nursing role of leadership, education, research and clinical practice with the client as its central focus.

Hepatology nursing is a recognized specialized area of nursing that focuses on the promotion of health, the prevention of illness, the care and support of clients experiencing liver-related diseases, and research. Key components include:

- Promotion of health - hepatology nurses perform activities that include providing educational sessions for the general public, marginalized populations and other health care professionals, as well as promoting harm-reduction initiatives such as safe needle disposal.

- Prevention of illness - activities include immunization, education regarding the prevention of the spread of disease and the needle exchange program.

- Care, support and treatment - nurses educate patients and their families; provide emotional support and advocacy; counsel both before, during and after treatment on benefits, risks, side effects, coping strategies and adherence to treatment; interpret results; and liaison with the family, support groups and other health professionals (ie, psychiatrist, ophthalmologist, social worker, etc).

- Research - nurses are active in clinical trials (industry and/or pharmaceutical), monitoring adherence to treatment, their own nurse-initiated studies, quality of life and continually incorporate new research- and evidence-based findings into their practice.

The hepatology nursing roles incorporate the activities of clinical practice, education, advocacy, counselling, collaboration, community support, leadership, administration and research.
From a recent survey completed by our membership, the hepatology nurse works with patients in a variety of settings including community or public health, street outreach clinics, methadone programs, correction facilities, private physicians' offices, hospitals and academic centres. Because of new developments in antiviral therapy for hepatitis, the hepatology nurses have become a vital part of the multidisciplinary team. The marginalized populations are complex, time consuming and resource intensive. The hepatology nurse is critical to their care and management. These dedicated nurses go the extra mile in providing support, education, counselling and referrals that provide the greatest opportunity for staying on treatment and receiving care.

In conclusion, Moirand et al have reported that only approximately $40 \%$ of the identified eligible patients in our inner-city HCV population actually receive treatment. Clearly, the proportions need to increase, and there are many challenges in the prevention, care and treatment for vulnerable populations. The multidisciplinary team approach, with the hepatology nurse playing an integral role, is a key strategy that can provide the opportunity for this population to receive care and management.

\section{REFERENCES}

1. Van Thiel DH, Anantharaju A, Creech S. Response to treatment of hepatitis $\mathrm{C}$ in individuals with a recent history of intravenous drug abuse. Am J Gastroenterol 2003;98:2281-8.

2. Sylvestre D. Hepatitis $C$ treatment in drug users: Perception versus evidence. Eur J Gastroenterol Hepatol 2006;18:129-30.

3. Sylvestre DL, Loftis JM, Hauser P, et al. Co-occurring hepatitis C, substance use, and psychiatric illness: Treatment issues and developing integrated models of care. J Urban Health 2004;81:719-34.

4. Backmund M, Meyer K, Von Zielonka M, Eichenlaub D. Treatment of hepatitis $\mathrm{C}$ infection in injection drug users. Hepatology 2001;34:188-93.

5. Canadian Association of Hepatology Nurses (CAHN). Hepatology nursing standards < www.cahn.ca> (Version current at January 23, 2007). 


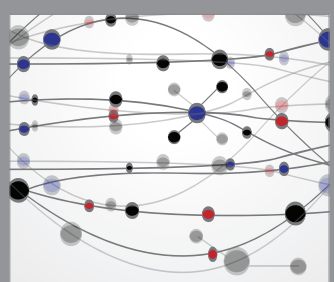

The Scientific World Journal
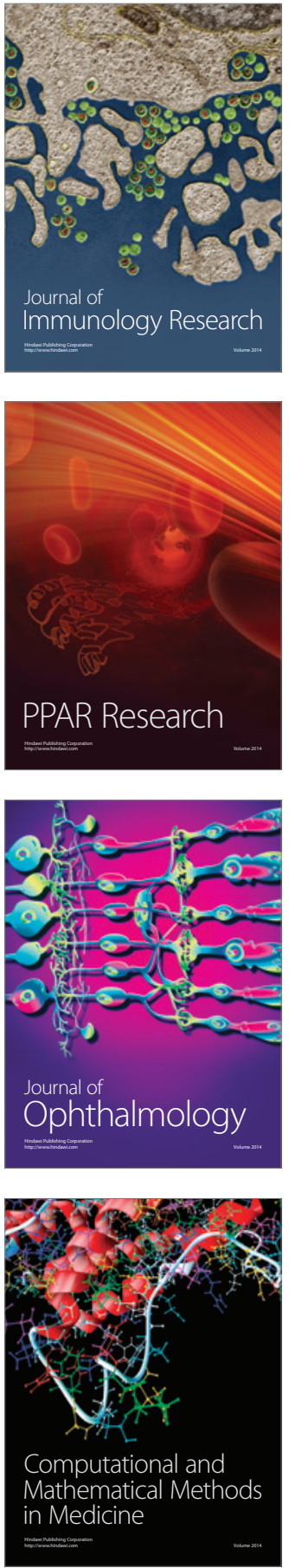

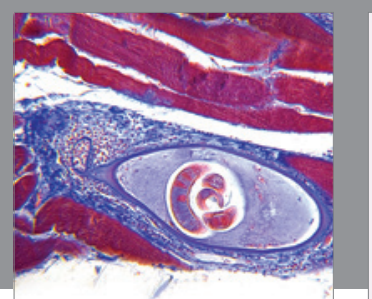

Gastroenterology Research and Practice

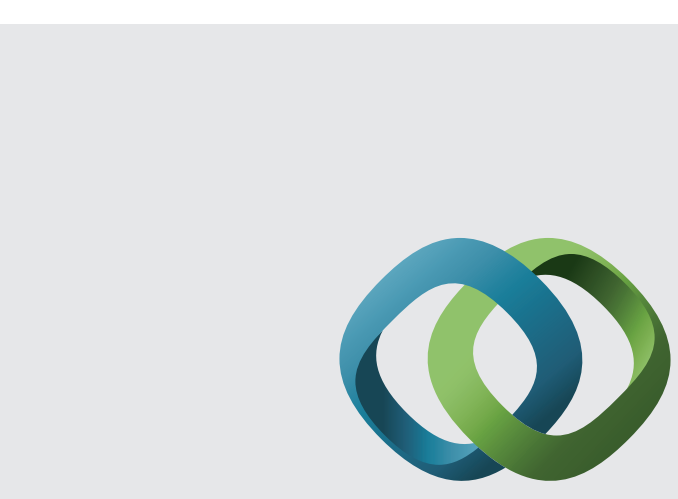

\section{Hindawi}

Submit your manuscripts at

http://www.hindawi.com
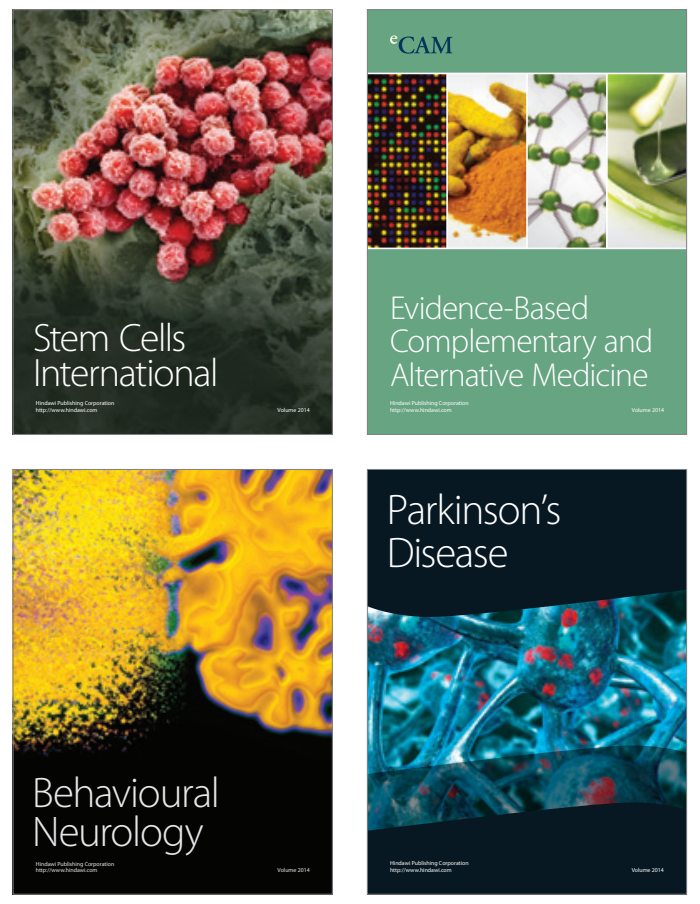
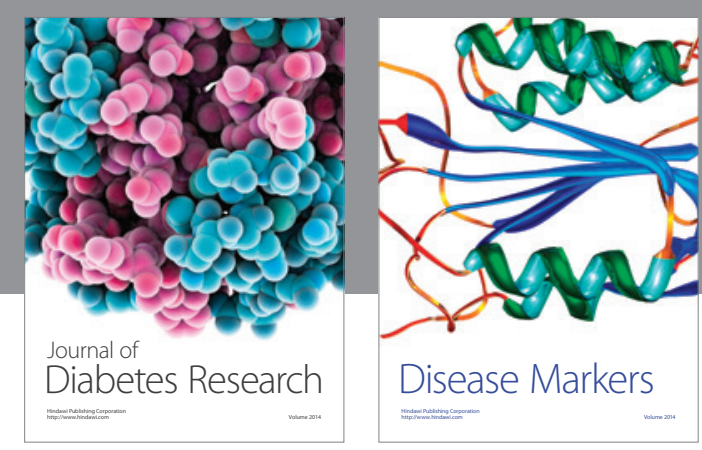

Disease Markers
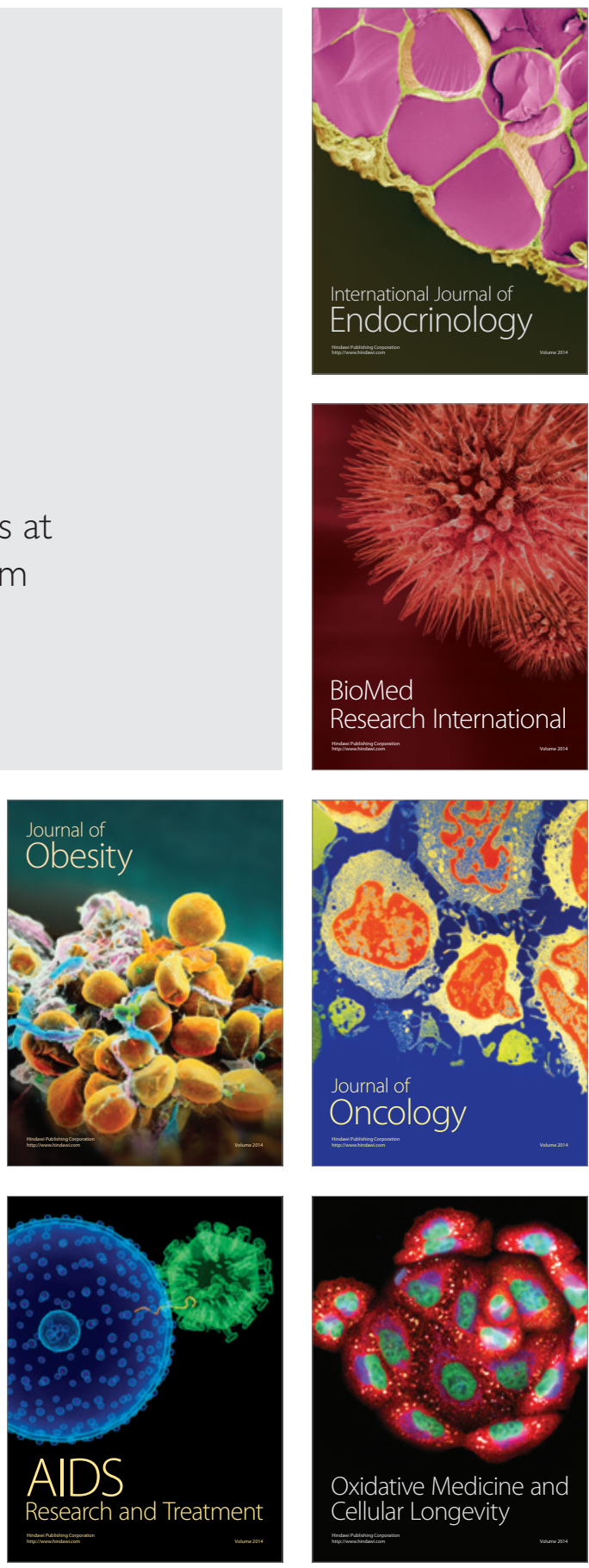